\title{
A Unique Case of Endovascular Repair of Aortic Aneurysm
}

Mohanty A*

DM Cardiology, Department of Cardiology, Continental Hospitals, Hyderabad, India

\begin{abstract}
A 57 year old asymptomatic male who is a smoker and a known case of Diabetes mellitus and hypertension was diagnosed as a case of suprarenal thoracoabdominal aortic aneurysm which included the origin of superior mesenteric artery (SMA) and celiac artery (CA). The origins of the CA SMA are occluded and are supplied retrogradely through extensive collaterals from the inferior mesenteric artery (IMA).

In thoracoabdominal aneurysms which include major side branches, usually we need to go for hybrid procedures or use of custom made branched stent grafts to maintain the patency of the side branches. This increases the operative time, operative complications as well as the cost of procedure. But in our patient, because of the occluded mesenteric arteries and extensive collaterals from IMA, we used a conventional graft stent across the aneurysmal segment covering the SMA and CA origin. Hence the operative time and procedural cost was significantly reduced by the 'natural bypass' which is revascularising the CA and SMA.
\end{abstract}

Keywords: Aortic aneurysm; Endovascular repair; Occluded mesenteric arteries

\section{Case Report}

A 57 year old male who is a smoker and a known case of Diabetes mellitus and hypertension underwent routine health check up which included ultrasonography (USG) of the abdomen. He was asymptomatic. His USG abdomen revealed aortic aneurysm of size $9.9 \mathrm{~cm}$ after which he was referred to the cardiovascular department. As part of workup we did a CT aortogram which showed a $9.9 \mathrm{~cm}$ suprarenal thoraco-abdominal aortic aneurysm of length $11 \mathrm{~cm}$ (Figure 1). The origin of the celiac artery (CA) and superior mesenteric artery (SMA) were occluded (Figures 2 and 3 ) and were supplied retrogradely through extensive collaterals from the inferior mesenteric artery (IMA). His right femoral and external iliac artery was showing mild calcification with plaques, but his left system was normal. He has single kidney (located on right side). His coronary angiogram did not show any significant lesion.

So after discussion with the 'Heart team', we decided to proceed with endovascular repair of the aneurysm. The stent graft planned for for the aneurysm would have covered the CA and SMA origin,

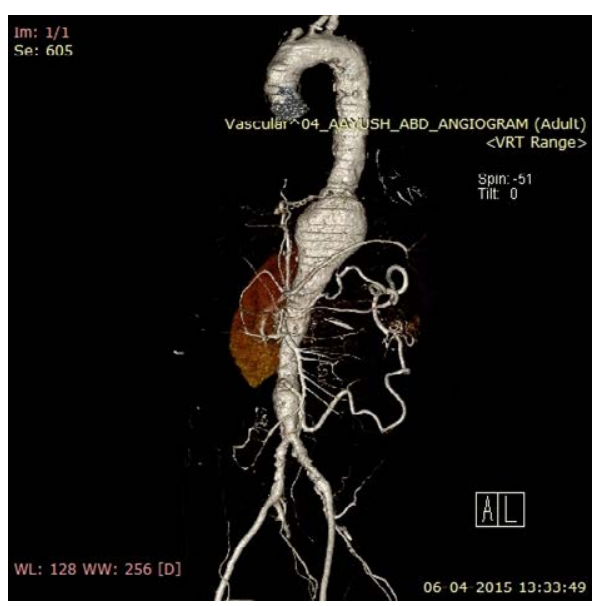

Figure 1: CT aortogram showing the thoraco abdominal aneurysm.

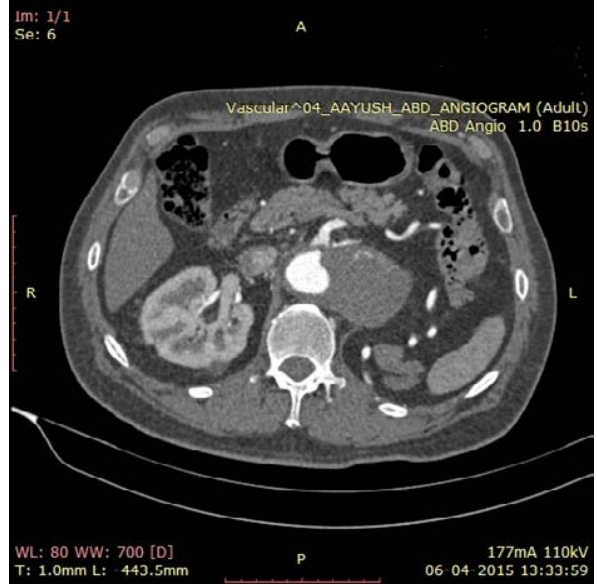

Figure 2: Axial view of ct aortogram showing occluded origin of superior mesenteric artery.

but since the origin of the above vessels were already occluded with extensive collateral from the IMA, we decided to go for a conventional self expanding stent rather than a branched stent graft.

The procedure was done successfully under general anaesthesia. The left femoral artery was accessed surgically $2 \mathrm{~cm}$ above the inguinal ligament. In the pre-operative angiogram we again marked the proximal and distal landing zones and confirmed the dimensions of the stent graft required (Figures 4 and 5). The post operative angiogram did

*Corresponding author: Mohanty A, DM Cardiology, Department of Cardiology, Continental Hospitals, Hyderabad, India, Tel: +91-9666121115 E-mail: abhisekh.mhnt@gmail.com

Received September 30, 2015; Accepted November 07, 2015; Published November 16, 2015

Citation: Mohanty A (2015) A Unique Case of Endovascular Repair of Aortic Aneurysm. J Vasc Med Surg 3: 232. doi:10.4172/2329-6925.1000232

Copyright: @ 2015 Mohanty A. This is an open-access article distributed under the terms of the Creative Commons Attribution License, which permits unrestricted use, distribution, and reproduction in any medium, provided the original author and source are credited. 


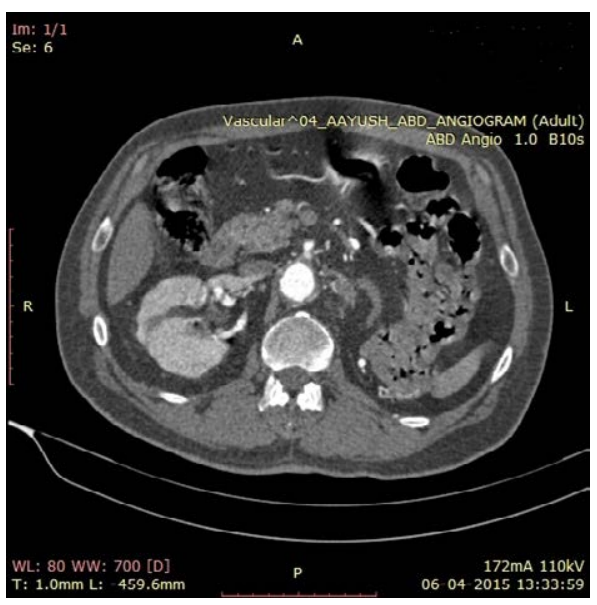

Figure 3: Axial view of ct aortogram showing the occluded origin of celiac artery.

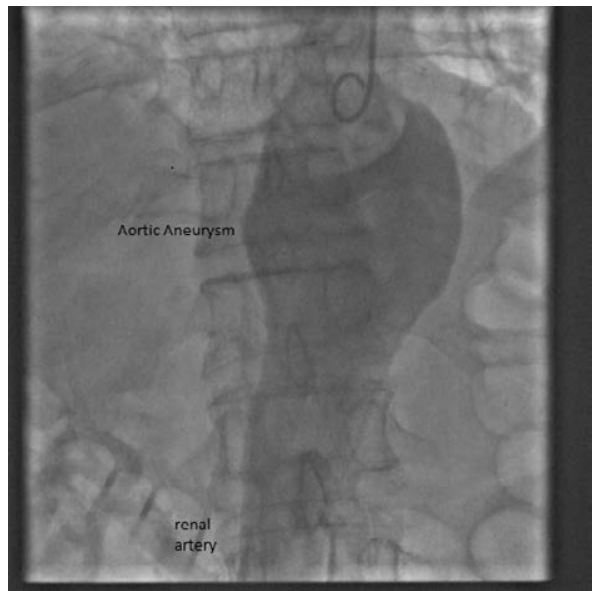

Figure 4: Pre-procedural aortogram showing the aortic aneurysm and the origin of the single renal artery.

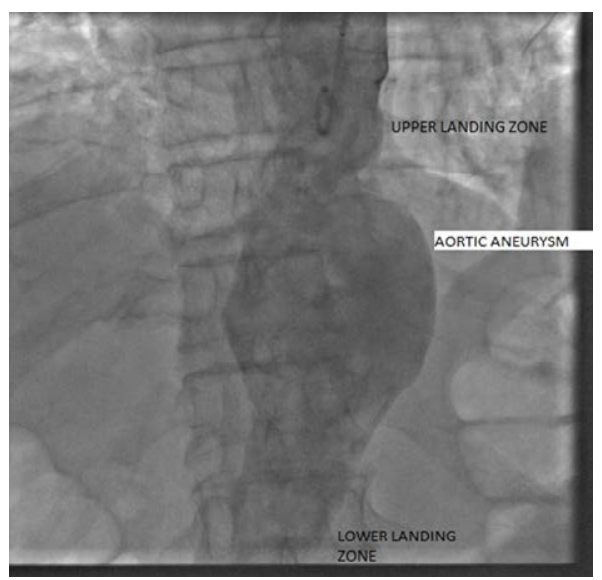

Figure 5: Pre-procedural aortogram showing the proximal and distal landing zones for the stent graft.

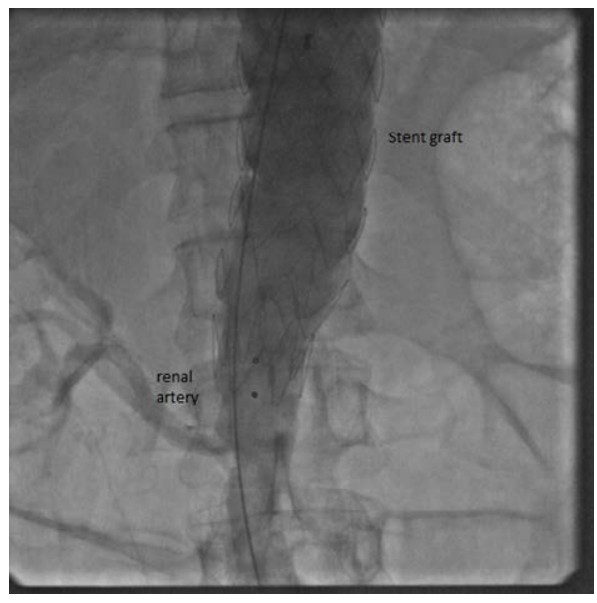

Figure 6: Post operative angiogram showing no endoleaks and preservation of the renal artery.

not reveal any endoleaks and the single renal artery was also preserved (Figure 6). The femoral artery was repaired and an angiogram was taken to demonstrate normal flow in the femoral artery.

EVAR is now the conventional management of aortic aneurysms with suitable anatomy [1]. This has been proven abundantly in registry and randomized trials, especially for infrarenal and descending thoracic aortic aneurysms. In case of thoraco - abdominal aortic aneurysms, EVAR is still in the evolving stage because of the presence of the important side branches of aorta which needs to be preserved. For this reason either we had to use custom made branched or fenestrated stent grafts [2] or use the 'chimney technique' [3]. Alternatively we have to do surgical revascularisation by doing bypass anastomosis, to secure the vessel before proceeding with EVAR.

Our case demonstrates how the natural bypass created by the extensive collaterals of IMA due to occlusion of CA and SMA simplified the procedure of endovascular repair. We were spared from using the branched stent grafts and also from surgery to create bypass anastomoses, which would have significantly increased the operative time, morbidity, chances of operative complications, as well as the cost of the procedure. The randomized trials like EVAR - 1, OVER and DREAM trials [4-6] comparing open versus EVAR, concluded that there is lower operative mortality, faster recovery, lower paraplegia incidence with EVAR. The rate of re-intervention was however found to be higher with EVAR because of stent graft failure. The long-term results from the OVER trial suggested a mortality 'catch-up' in the EVAR group after 3 years [5].

\section{References}

1. Brown LC, Powell JT, Thompson SG, Epstein DM, Sculpher MJ, et al. (2012) The UK EndoVascular Aneurysm Repair (EVAR) trials: randomised trials of EVAR versus standard therapy. Health Technol Assess 16: 1-218.

2. Ferreira M, Lanziotti L, Cunha R, d'Utra G (2012) Thoracoabdominal aortic aneurysm: a totally endovascular approach with a branched stent-graft. Ann Cardiothorac Surg 1: 406-408.

3. Moulakakis KG, Mylonas SN, Avgerinos E, Papapetrou A, Kakisis JD, et al. (2012) The chimney graft technique for preserving visceral vessels during endovascular treatment of aortic pathologies. J Vasc Surg 55: 1497-1503.

4. EVAR trial participants (2005) Endovascular aneurysm repair versus open repair in patients with abdominal aortic aneurysm (EVAR trial 1): randomised controlled trial. Lancet 365: 2179-2186. 
5. Lederle FA, Freischlag JA, Kyriakides TC, Padberg FT Jr, Matsumura JS, et al. (2009) Outcomes following endovascular vs open repair of abdominal aortic aneurysm: a randomized trial. JAMA 302: 1535-1542.
6. De Bruin J, Baas A, Buth J (2010) Long-term outcome of open or endovascular repair of abdominal aortic aneurysm. N Engl J Med 362: 1881-1889. 